\title{
The Effect of Time-Delay Feedback Controller on an Electrically Actuated Resonator
}

\author{
S. Shao, K. M. Masri, and M.I. Younis \\ Binghamton University, State University of New York PO BOX 6000 Binghamton, NY 13902-6000, USA. \\ myounis@binghamton.edu
}

\begin{abstract}
This paper presents a study of the effect of a time-delay feedback controller on the dynamics of a Microelectromechanical systems (MEMS) capacitor actuated by DC and AC voltages. It is shown that negative time-delay feedback control gain can lead to an unstable system, even if AC voltage is relatively small compared to DC voltage. Perturbation method is utilized to present analytically the nonlinear dynamic characteristics of the MEMS capacitor. Agreements among the results of a shooting technique, long-time integration, basin of attraction analysis with the perturbation method are achieved.
\end{abstract}

\section{Introduction}

Delay in MEMS devices is a very common phenomenon, which can be introduced into the system unavoidably or by design. The desire of improved device features, such as low-cost, low-voltage, high quality factor, and improved reliability has motivated great interest recently to understand the impact of delays on MEMS.

For electrostatic MEMS resonators, many inherent system delays can be introduced through actuators, filters, processor dynamics, and feedback measurements. Feedback controllers are applied to stabilize the response, compensate for system parameter changes, and generally to enhance the resonator performance. Especially challenging is to drive electrostatic MEMS resonator at sharp response while preventing them from collapse due to pull-in.

Recent years have witnessed numerous studies on the time-delay systems and their application. An effective method of oscillators stabilization has been developed by Pyragas [1], which was originally proposed to stabilize the unstable periodic orbits of a chaotic system. Wang \& $\mathrm{Hu}$ [2] applied several singular perturbation methods, such as methods of multiple scales and averaging, to model a controlled Duffing oscillator with delayed velocity feedback. They also applied other methods including the Lyapunov function for stability combined with averaging, the energy analysis, and pseudo-oscillator analysis [3].

In a previous work, Younis and Nafyeh [4] used a perturbation method to analytically describe dynamics of a resonant microbeam excited electrically [4]. Alsaleem and Younis [5] investigated theoretically the dynamics of MEMS resonators using shooting technique and basin of attraction analysis and verified their results experimentally.

In this paper, we use a single-freedom model to investigate the dynamics of electrostatic MEMS resonators with the delayed feedback controller of [1]. A perturbation method, the method of multiple scales, is used to present analytically the impact on the dynamic system by the control gain. The results are then verified using long-time integration, shooting techniques, and basin of attraction analysis.

\section{Problem Formulation}

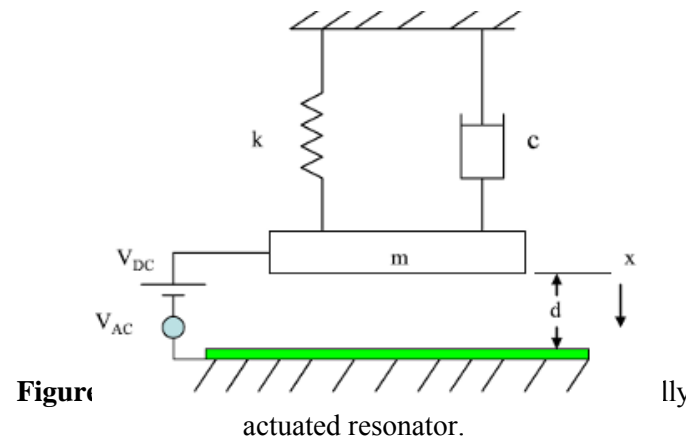

We consider a nonlinear single-degree-of-freedom model (Fig. 1) actuated by an electric load composed of a DC component $V_{D C}$ and an AC harmonic component $V_{A C}$ subject to a viscous damping of coefficient $c$. The equation of motion governing the behavior of the resonator under a delay feedback controller can be expressed as 


$$
\begin{aligned}
& m \frac{d^{2} u}{d t^{2}}+c \frac{d u}{d t}+k(u+\delta)= \\
& \frac{\varepsilon_{0} A\left[V_{D C}+V_{A C} \cos \Omega t+G\left(\dot{u}_{d}-\dot{u}\right)\right]^{2}}{2(d-\delta-u)^{2}}
\end{aligned}
$$

where $\delta$ is the equilibrium position of the oscillator due to $V_{D C}, u$ is the dynamic amplitude of the motion $(x=u+\delta)$, $\varepsilon_{0}$ is the dielectric constant of air, $A$ is the electrode area, $\Omega$ is the AC excitation frequency, $G$ is the amplitude of the controller, and $\dot{u}_{d}=\dot{u}(t-\tau)$, where $\tau$ is the time delay. The rest of parameters are as shown in Fig. 1. Next, we normalize the equation by introducing the nondimensional variables

$$
\tilde{t}=\frac{t}{T}, \tilde{\tau}=\frac{\tau}{T}, \tilde{u}=\frac{u}{d}, \tilde{u}_{d}=\frac{u_{d}}{d}, \tilde{\delta}=\frac{\delta}{d},
$$

where $T=\sqrt{m / k}$. After expanding the electrostatic force term up to the third order, we end up with the below nondimensional equation

$$
\ddot{\tilde{u}}+2 \zeta \dot{\tilde{u}}+\omega^{2} \tilde{u}+\alpha_{q} \tilde{u}^{2}+\alpha_{c} \tilde{u}^{3}=2 f \cos \tilde{\Omega} \tilde{t}+2 g \dot{\tilde{u}}_{d}
$$

where $\zeta=\frac{c}{2 \sqrt{m k}}+\frac{P V_{D C} \tilde{G}}{(1-\tilde{\delta})^{2}}, \omega^{2}=1-\frac{2 P V_{D C}^{2}}{(1-\tilde{\delta})^{3}}$,

$\alpha_{q}=-\frac{3 P V_{D C}^{2}}{(1-\tilde{\delta})^{4}}, \alpha_{c}=-\frac{4 P V_{D C}^{2}}{(1-\tilde{\delta})^{5}}, f=\frac{P V_{D C} V_{A C}}{(1-\tilde{\delta})^{2}}$,

$g=\frac{P V_{D C} \tilde{G}}{(1-\tilde{\delta})^{2}}, P=\frac{\varepsilon_{0} A}{2 k d^{3}}, \tilde{\Omega}=\Omega T, \tilde{G}=\frac{G d}{T}$.

Applying the method of multiple scales [6] on Eq. (2) yields the following modulation equations governing the amplitude $a$ and phase $\varphi$ of the dynamic response:

$$
\begin{aligned}
& a^{\prime}=-\zeta a+\frac{f}{\omega} \sin \varphi+g a \cos (\omega \tau) \\
& a \varphi^{\prime}=a \sigma+\left(\frac{5 \alpha_{q}^{2}}{12 \omega^{3}}-\frac{3}{8 \omega} \alpha_{c}\right) a^{3}+\frac{f}{\omega} \cos \varphi+g a \sin (\omega \tau)
\end{aligned}
$$

Based on Eqs. (3) and (4), the steady-state frequencyresponse equation can be written as

$$
\begin{aligned}
& a^{2}\left\{\left[\left(\frac{5 \alpha_{q}^{2}}{12 \omega^{3}}-\frac{3 \alpha_{c}}{8 \omega}\right) a^{2}+\sigma+g \sin (\omega \tau)\right]^{2}+\right. \\
& \left.[g \cos (\omega \tau)-\zeta]^{2}\right\}=\left(\frac{f}{\omega}\right)^{2}
\end{aligned}
$$

From Eq. (5) we can see the term $g \sin (\omega \tau)$ plays the role of varying the excitation frequency $\sigma$, since $\sigma$ represents the deviation from the natural frequency. Similarly, $g \cos (\omega \tau)$ plays the role of varying the effective damping. Usually, $\tau=T / 2=\pi / \omega$ is adopted, and then the effect of gain can be described as $|g-\zeta|$.

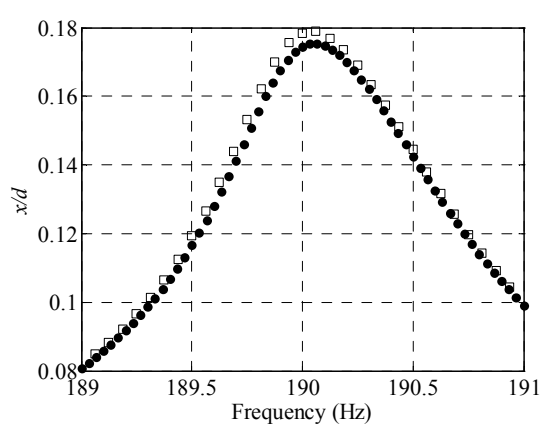

Figure 2. Analytical and simulation results for the frequency response for $G=0, V_{A C}=1 \mathrm{~V}$, and $V_{D C}=40.2 \mathrm{~V}$ (squares: longtime integration; points: perturbation).

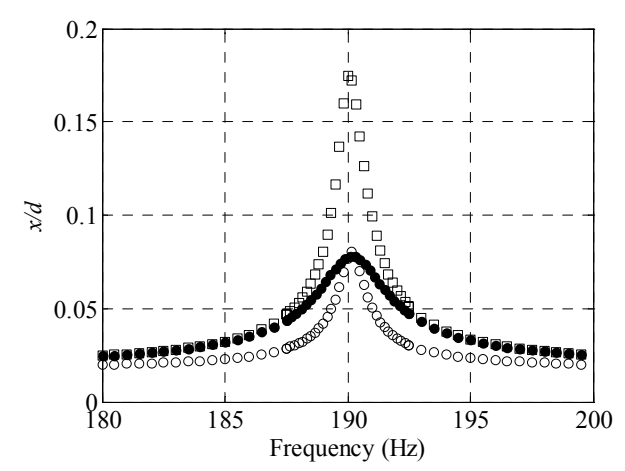

Figure3. Positive gain versus $\mathrm{AC}$ voltage effect for $V_{D C}=40.2$ $\mathrm{V}$ and $\tau=T / 2$ (squares: $V_{A C}=1 \mathrm{~V}, G=0$; circles: $V_{A C}=0.6 \mathrm{~V}$, $G=0$; points: $\left.V_{A C}=1 \mathrm{~V}, G=110 \mathrm{Vs} / \mathrm{m}\right)$.

Next, we use Eq. (5) to analytically generate frequencyresponse curves of the resonator for various values of gain and voltage loads. Also, we will compare these results to results of direct time integration of Eq. (1)

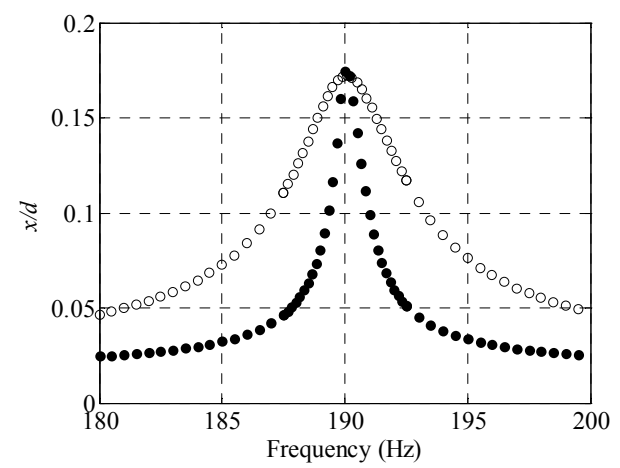

Figure 4. Comparison of the effect of using a positive gain versus $V_{A C}$ to yield the same dynamic amplitude (circles: $V_{A C}$ $=3.8 \mathrm{~V}, \mathrm{G}=200 \mathrm{Vs} / \mathrm{m}$; points: $\left.V_{A C}=1 \mathrm{~V}, \mathrm{G}=0\right)$.

\section{Result and analysis}

This section presents results based on the capacitive resonator studied in [5]. The resonator is composed of two long cantilever beams attached to a proof mass at the tip. First, we show results for an un-controlled case with small voltage loads. Figure 2 compares the frequency response curve (normalized response versus frequency) obtained by the method of multiple scales (MMS) to that 
of long-time integration of Eq. (1). As shown, the agreement is good among the results. Figure 3 demonstrates the effect of applying a control gain using the MMS. First we show the solution for $V_{A C}=1 \mathrm{~V}$ with no gain. Decreasing $V_{A C}$ lowers the amplitude response, shown in circles in the figure. We can see however that the response bandwidth shrinks. On the other hand, applying a control gain instead of lowering the excitation voltage, one can see the positive gain not only does it reduce the response amplitude, but also it keeps the bandwidth unchanged. Figure 4 demonstrates another way of taking advantage of this dynamical feature by comparing uncontrolled response of the resonator to a controlled response using lower values of $V_{A C}$ such that both actuation methods yield the same maximum response amplitude. Clearly, the controlled response has much wider bandwidth. This remarkable result can be of great advantage in MEMS for sensing and energyharvesting applications, where both sharp response and wide bandwidth are desirable.

Next, we investigate the effect of negative gain on the dynamics. We establish a Jacobin matrix based on Eq. (3) and Eq. (4) and solve for eigenvalues to judge the system's stability.

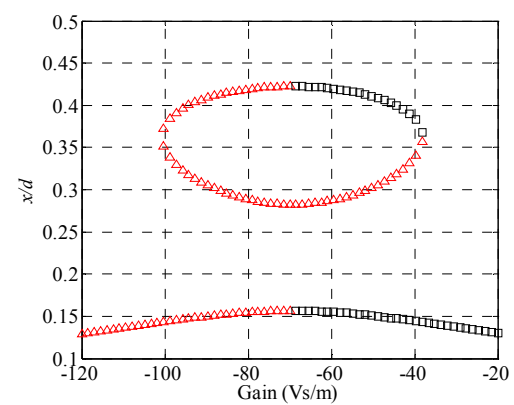

Figure 5. Gain sweeping result at frequency $=189.5 \mathrm{~Hz}$ for $V_{A C}$ $=1 \mathrm{~V}$ and $V_{A C}=40.2 \mathrm{~V}$ (squares: stable; triangles: unstable).

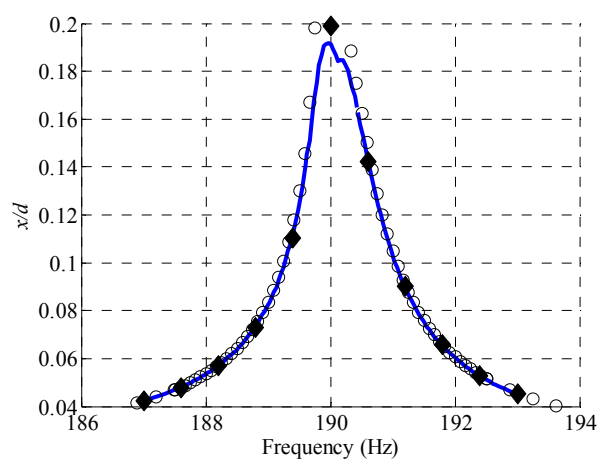

(a) $G=-20 \mathrm{Vs} / \mathrm{m}$

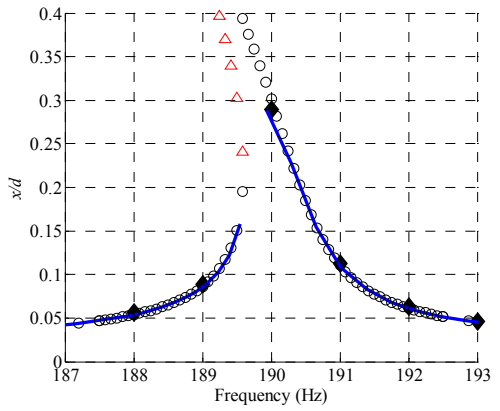

(b) $G=-50 \mathrm{Vs} / \mathrm{m}$

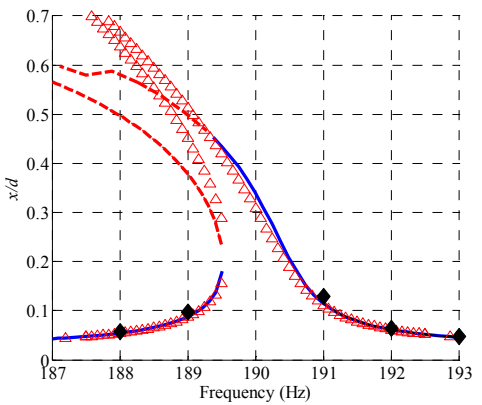

(c) $G=-80 \mathrm{Vs} / \mathrm{m}$

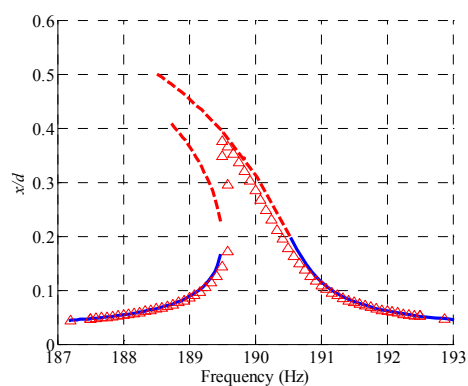

(d) $G=-100 \mathrm{Vs} / \mathrm{m}$

Figure 6. Comparison between Perturbation method (MMS) and shooting technique results as well as time integration separately. Time delay is $T / 2$. Same $V_{A C}$ and $V_{D C}$ as above (circles: perturbation stable; triangles: perturbation unstable; solid: shooting stable; dashed: shooting unstable; diamonds: long time integration).

Figure 5 shows bifurcations occur within the interval $G=-40 \sim-70 \mathrm{Vs} / \mathrm{m}$. Figure 6 , a set of frequency response curves with different gains illustrating the scenario of Fig. 5 in another perspective. In Fig. 6a, consider the case of $G=-20 \mathrm{Vs} / \mathrm{m}$. The response curve has a linear shape with no hysteresis. Thus, at the excitation frequency of 189.5 $\mathrm{Hz}$, it has one single value of a stable response. When increasing the gain to $G=-50 \mathrm{Vs} / \mathrm{m}$ (Fig. 6b), the response becomes of softening behavior with hysteresis. Thus, at $189.5 \mathrm{~Hz}$, the response can be a stable of low value, unstable, or stable at higher value. Increasing the gain further to $G=-80 \mathrm{Vs} / \mathrm{m}$ (Fig. $6 \mathrm{c}$ ), according to the perturbation results, the frequency-response curve becomes entirely unstable.

Using a shooting technique to find periodic motions combined with the Floquet theory, we found good agreement with the perturbation results for small values of gain. For larger values of negative gain, shooting predict some regions of stable response while 
perturbation predicts unstable solutions everywhere. According to the shooting results, a pair of Floquet multiplier exists the unit circle through complex numbers, which indicate a secondary Hopf bifurcation.

Next, we analyze the basin of attraction of the obtained solution for the specific operating point of $\Omega$ $=189.5 \mathrm{~Hz}$ for the various gains of Fig. 6 . The basin of attraction is obtained by integrating the equation of motion, Eq. (1), in time for various initial conditions using a grid of 500x500 initial velocity and displacement. We use the special subprogram in MATLAB, DDE23, for delayed differential equations [7]. We assume the initial state of the system to be constant (equal to the initial condition) prior to the application of the delayed signal.

In the case of $G=-50 \mathrm{Vs} / \mathrm{m}$, Fig. $7 \mathrm{a}$, one can see that there is a large safe area. As the negative value of $G$ is increased to $G=-80 \mathrm{Vs} / \mathrm{m}$, the safe area erodes leading to a very small basin of attraction, Fig. 7b. Practically speaking, the resonator is most likely to pull-in in this case. It supports the MMS result in the perspective of the system's initial states. However, this partly matches shooting technique results locally near $190 \mathrm{~Hz}$ (natural frequency). One should note that the perturbation method analyzes the response locally near primary resonance in the neighborhood of natural frequency. Increasing the negative value of $G$ further to $G=-100 \mathrm{Vs} / \mathrm{m}$ leads to complete erosion of the basin of attraction, Fig. 7c.

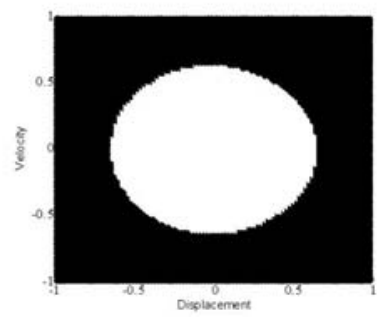

(a) $G=-50 \mathrm{Vs} / \mathrm{m}$

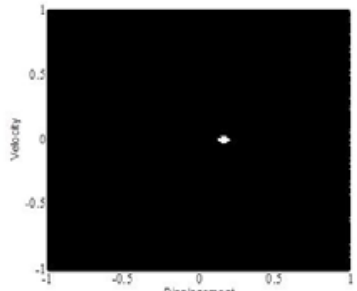

(b) $G=-80 \mathrm{Vs} / \mathrm{m}$

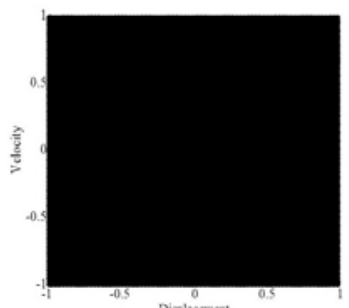

(c) $G=-100 \mathrm{Vs} / \mathrm{m}$

Figure 7. Basin of attractions for different negative gains at excitation frequency $=189.5 \mathrm{~Hz}$ (white areas represent initial conditions leading to stable solution).

\section{Summary and conclusions}

This paper presented analytical solution for a singledegree-freedom resonator model actuated by $V_{D C}$ and $V_{A C}$ with a delay feedback controller. We used method of multiple scales (MMS) to obtain an analytical explanation for the effect of control gain. The MMS results for nonlinear dynamic system with no controller agree well with the long-time integration results. For negative control gain, MMS presents bifurcation behavior, showing that the system reaches unstable state when gain is negative enough even though the harmonic load $V_{A C}$ is relatively small (for example $V_{A C}=1 \mathrm{~V}, V_{D C}$ $=40.2 \mathrm{~V}$ ). This nonlinear property can lead to an attractive industry application, such as low-actuation voltage MEMS switches.

The analytical results indicate another attractive feature in the case of positive gains to achieve sharp response of resonators while maintaining large bandwidth. This can be also very attractive for variety of sensing and actuation application in MEMS.

We compared the results of the perturbation method to those of the shooting technique and the basin of attraction analysis. The basin of attraction indicated that while shooting predicts stable regime in the frequencyresponse curves for large values of negative gain; the basin of attraction of these states are extremely small and fragile. This surprisingly agrees with the analytical results from MMS, which predicts unstable solutions everywhere. Moreover, the shooting results predict secondary Hopf bifurcation for some large values of negative gain. This may lead to quasi-periodic motion as well as other complex dynamic behaviors.

\section{References}

1. Pyragas, K. (1992). Continuous Control of Chaos by Self-Controlling Feedback. Physics Letters A 170(6): 421-428.

2. Hu, H. Y. and Z. H. Wang (2009). Singular perturbation methods for nonlinear dynamic systems with time delays. Chaos Solitons \& Fractals 40(1): 13-27.

3. Hu, H. Y., H. L. Wang, et al. (2004). Global dynamics of a Duffing oscillator with delayed displacement feedback. International Journal of Bifurcation and Chaos 14(8): 2753-2775

4. Younis, M. I. and A. H. Nayfeh (2003). A study of the nonlinear response of a resonant microbeam to an electric actuation. Nonlinear Dynamics 31(1): 91-117.

5. Alsaleem, F. M. and M. I. Younis (2010). Stabilization of electrostatic MEMS resonators using a delayed feedback controller. Smart Materials \& Structures 19(3).

6. Nayfeh, A. H., "Introduction to perturbation techniques," Wiley, 1981, New York.

7. www.mathworks.com/help/techdoc/ref/dde23.ht $\mathrm{ml}$ 